\title{
Pseudopterosin A ameliorates ischaemia-induced brain injury by acting on Akt signalling pathway
}

\author{
Xinkai Niu ${ }^{1}$, Chaochun $\mathrm{Yu}^{2}$, Guanfu Jiang ${ }^{2}$, Jun $\mathrm{Wu}^{2}$, Xiaohui $\mathrm{Tan}^{2}$, Weijia Zhang ${ }^{2}$, Lijun $\mathrm{Hou}^{1}$ \\ ${ }^{1}$ Department of Neurosurgery, Second Military Medical University affiliated with Changzheng Hospital, Shanghai, ${ }^{2}$ Department \\ of Neurosurgery, Shanghai University of Traditional Chinese Medicine affiliated with Shanghai TCM Integrated Hospital, Shanghai, \\ China
}

\begin{abstract}
Introduction: Brain injury caused by ischaemic stroke is a major cause of disability and death throughout the world. The present study evaluates the neuroprotective effect of pseudopterosin A (PtA) against ischaemia-induced brain injury.

Material and methods: Ischaemia was induced by PMCAO model, and rats were separated in to three groups. A control group; an ischemic group receiving saline solution; and a PtA group receiving PtA (5 mg/kg, i.p.) for the period of three days, i.e. two days before surgery and after the surgery. Effect of PtA was assessed by estimating the infract volume and neurological deficits. Level of oxidative stress, inflammatory mediators, and markers of apoptosis were estimated in the brain tissues. Western blot assay and immunohistochemistry was done for the assessment of expressions of protein.

Result: Data from the study suggest that treatment with PtA significantly decreases the infracted volume and neurological deficit score compared to the ischaemic group. Treatments with PtA attenuate the activity of antioxidant enzyme, and the level of inflammatory mediators and markers of apoptosis in the brain tissues of ischaemia-induced brain injury. Phosphorylation of FKHR, Bad, and Akt were significantly reduced in the PtA-treated group compared to the ischaemic group.

Conclusions: The present study shows that pseudopterosin A attenuates neuronal injury in the PMCAO model by acting on the Akt signalling pathway.
\end{abstract}

Key words: pseudopterosin A, brain injury, Akt signalling, apoptosis.

\section{Introduction}

Brain injury caused by ischaemic stroke is a major cause of disability and death throughout the world [4]. The pathophysiology of ischaemic stroke is complex and so is its therapy. Injury occurring due to ischaemia cannot be completely healed regard- ing the function of tissue and organs. Haemorrhagic transformation, cerebral oedema, and neuronal death-like events occur in brain damage due to cerebral ischaemia [16]. There are several mechanisms involved in the pathogenesis of brain injury, such as disturbance of blood brain barrier, excitotoxicity, oxidative stress, activation of apoptosis, and inflam- 
matory mediators [18]. The literature reveals that apoptosis and inflammatory response are regulated by Akt and phosphoinositide 3-kinases (PI3Ks) in ischaemic brain injury [11]. In injury induced by ischaemia activation of downstream protein is achieved due to enhanced expression of p-Akt protein. Several reports reveal that the agent that alters the expressions of $\mathrm{p}$-Akt attenuates the neuronal injury by reducing the inflammation and apoptosis of neuronal cells $[19,12]$. Management of stroke is still a challenge, and the drug used for the treatment of stroke has some serious side effects, such as neurotoxicity. Thus, more focus has been placed the development of alternative medicine that can act on the pathogenic pathway of brain injury.

Pseudopterosin A (PtA) is isolated from Pseudopterogorgia elisabethae (gorgonian sea whip) chemically known as diterpene glycoside [8]. Literature reveals that around 31 pseudopterosin derivatives have been identified and isolated from natural origins [13]. Cytotoxic and biological activities of pseudopterosins depend upon the type of sugar and glycosylation position on the terpene skeleton [3]. Pseudopterosins reported to possess several activities such as anti-tuberculosis, anti-malarial, anti-viral, anti-cancer, wound-healing, and anti-inflammatory activity $[14,15,20]$. Thus the present study evaluates the protective effect of pseudopterosin A against ischaemic/reperfusion-induced brain injury.

\section{Material and methods}

\section{Animal}

Male albino Wistar rats (120-150 g) were procured from Shanghai medical college, Shanghai, China. Animals were stored under the standard condition according to the guidelines. All the animals were kept for a period of seven days for acclimatisation to laboratory conditions with ad libitum access to normal standard chow diet and tap water. All the protocols were approved by the Institutional Animal Ethical Committee of the Second Military Medical University affiliated with Changzheng Hospital, China (IAEC/SMMU/CH/2017/02).

\section{Induction of brain injury}

Brain injury was induced by ischaemia produced using the PMCAO model according to the previously reported method. Isolation of the carotid artery was performed, and blood flow was reduced by inserting $20 \mathrm{~mm}$ of mono filament into it. The body temperature of the animals was maintained at $37^{\circ} \mathrm{C}$ during the period of surgery and the flow of blood was monitored. In the control group a filament was not inserted, but the surgical procedure was carried out in the same way as described earlier [10].

\section{Protocol design}

Pseudopterogorgia elisabethae was used for the isolation of pseudopterosin A (PtA) after collecting it from Burrows Cay, Bahamas. Isolation of PtA was performed according to the previously reported method [9]. Pseudopterosin A was dissolved in saline solution and administered intraperitoneally for period of three days, i.e. two days before and one day after the surgery, at a dose of $5 \mathrm{mg} / \mathrm{kg}$. All the animals were separated in to three groups $(n=10)$. A control group receiving saline without ischaemia; an ischemic group receiving saline solution; and a PtA group receiving PtA (5 mg/kg, i.p.).

\section{Evaluation of neurological function}

The neurological deficit score was estimated according to the previously reported method. Behaviour was estimated for the neurological deficit score, and the score was graded on the following scale: no neurological defects -0 ; flexion of forelimb -1 ; failure to extend right forelimb and decrease in the strength - 2; impairment of movement towards the right side, such as crawling and rotating - 3; difficulty in spontaneous movement -4 .

\section{Evaluation of infracted volume}

All the animals were sacrificed by decapitation method and brain was isolated from each animal. Isolated brain was cut into sections, and 2,3,5-triphenyltetrazolium chloride (TTC) was used to stained the tissue section for $15 \mathrm{~min}$. Image Pro-Plus 5.1 software was used for the estimation of the infracted area of the brain.

\section{Estimation of oxidative stress}

Antioxidant enzymes such as catalase (CAT) and superoxide dismutase (SOD) were measured in the tissue homogenate of brain tissues by using commercial kits. The procedure was used for the deter- 
mination of antioxidant enzymes according to the direction given by the manufacturer.

\section{Estimation of inflammatory mediators and markers of apoptosis}

The Nuclear/Cytosolic Fractionation Kit was used for the isolation of nuclear factor $\kappa B$ (NF- $\kappa B$ ) free p 65 subunit and the ELISA kit was used for its determination. ELISA method was used for the determination of level of caspase- 3 and tumour necrosis factor- $\alpha$ (TNF- $\alpha$ ) using their respective kits according to the manufacturer's directions.

\section{Western blot assay}

Cerebral cortex was dissected from the isolated brains and buffer containing 1 mM EDTA and Triton X-100 (1\%) in 7.4 pH PBS was used for the lysis of brain tissues. Brain tissue was centrifuged for the period of 20 min at $12000 \times$ at a temperature of $4^{\circ} \mathrm{C}$. Then the concentration of protein was estimated in the lysate in the collected supernatant solution using the bicinchoninic acid (BCA) kit according to the directions given by the manufacturer. SDS-polyacrylamide gels (10\%) was used to apply total protein on each lane, and after washing the membrane with Tris-buffer saline it was incubated with primary antibodies such as anti-cleaved-caspase-3, antiFKHR, anti-phospho-FKHR, anti-Bad, anti-phosphoBad, anti-Akt, anti-phospho-Akt, and anti- $\alpha$ tubulin. Further secondary antibody was used to incubate the membrane. Manufacturer's directions were used for application of the ECL Western blot analysis sys-

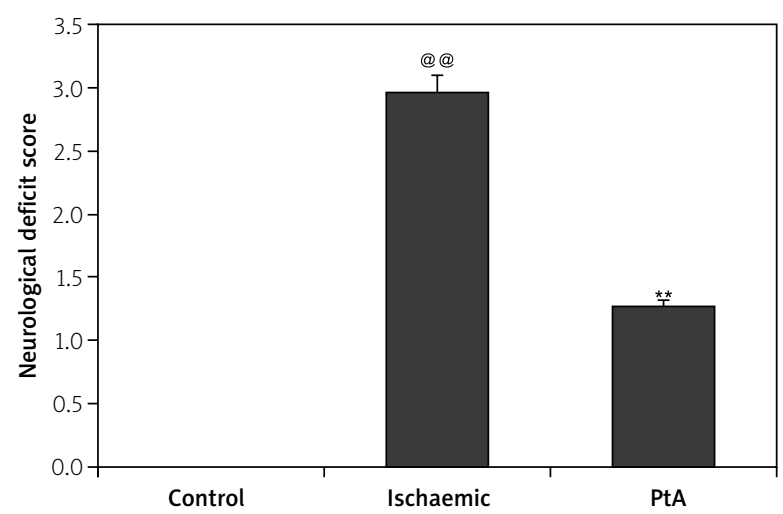

Fig. 1. Effect of pseudopterosin A (PtA) on the neurological deficit score in ischaemia-induced brain-injured rats. tem. Sigma Plot 4.0 and Sigma Gel 1.0 were used to estimate the intensity of western blot.

\section{Immunohistochemistry}

Paraformaldehyde (4\%) was used for the fixation of brain tissues, and then tissues were seeded in the molten paraffin. Tissues were cut in to section of 7-mm thickness using microtome. Section tissues were stained with antibody of Akt for a period of $60 \mathrm{~min}$. Anti-goat lgG was used to incubate the tissue section for the duration of 60 min after washing. Tissue sections were further washed and again incubated for the period of $60 \mathrm{~min}$ with anti-ionised calcium-binding adapter molecule 1. Moreover, FITC-conjugated anti-rabbit was incubated for the period of $60 \mathrm{~min}$ after washing the tissues, and fluorescence mounting medium was used to cover the section. A fluorescence microscope was used to view the tissue sections.

\section{Statistical analysis}

All data were expressed as mean \pm SD $(n=10)$. The statistical analysis was performed using oneway ANOVA. Post-hoc comparison of means was carried out by Dunnett's post hoc test (Gradpad prism 6.1., CA, USA). The level of statistical significance was set at $p<0.05$.

\section{Result}

\section{The effect of pseudopterosin A on neurological function}

Effect of PtA on the neurological deficit score in ischaemia-induced brain-injured rats is shown in Figure 1. Neurological deficit score was estimated by scaling the behavioural parameters. In the ischaemic group of rats the neurological deficit score $(2.95$ $\pm 0.15)$ was significantly enhanced compared to the control group of rats. However, treatment with PtA significantly decreased by up to $1.27 \pm 0.05$ compared to the ischaemic group.

\section{The effect of pseudopterosin A on cerebral infract}

Effect of PtA on the percentage of infract volume in the brain of ischaemia-induced brain-injured rats is shown in Figure 2. It was observed that the percentage of infract volume was significantly enhanced in the brain of ischaemia-induced brain-injured rats 
(ischaemic group) by up to $41.7 \%$ compared to the control group, while the percentage of infract volume was found to be reduced (12.9\%) in the tPtA-treated group compared to ischaemic group.

\section{The effect of pseudopterosin A on anti-oxidant enzymes}

The effect of PtA on the activity of anti-oxidant enzymes in the brain tissues of ischaemia-induced brain-injured rats is shown in Figure 3. There was a significant decrease in the activity of anti-oxidant enzymes such as CAT and SOD in the brain tissues of the ischaemic group, whereas treatment with PtA significantly attenuated the altered level of antioxidant enzyme in the brain tissues of ischaemiainduced brain-injured rats.

\section{The effect of pseudopterosin A on apoptosis and inflammatory markers}

The effect of PtA on markers of apoptosis and inflammatory mediators was assessed in the brain tissues of ischaemia-induced brain-injured rats, as shown in Table I. In the ischaemic group of rats the levels of markers of apoptosis, i.e. caspase- 3 and inflammatory mediators - NF- $\mathrm{kB}$ p65 and TNF- $\alpha$, was significantly enhanced $(p<0.01)$ in the brain tissues of ischaemia-induced brain-injured rats compared to the control group. However, treatment with PtA attenuates the altered level of markers of apoptosis and inflammatory mediators in brain tissues of ischaemia-induced brain-injured rats.
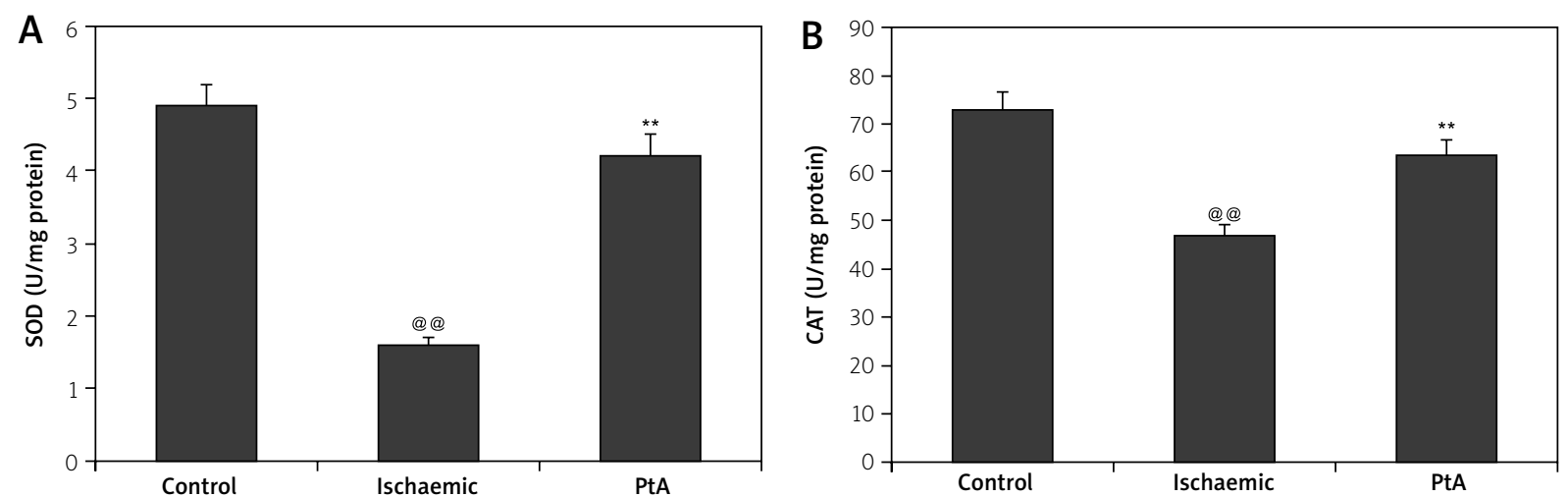

Data mean \pm SD $(n=10)$

(@) $p<0.01$ compared to the control group; ${ }^{* *} p<0.01$ compared to the ischaemic group

Fig. 3. Effect of pseudopterosin A (PtA) on the activity of anti-oxidant enzymes: A) superoxide dismutase (SOD), B) catalase (CAT), in the brain tissues of ischaemia-induced brain-injured rats. 
Table I. Effect of pseudopterosin A (PtA) on the level of apoptosis and inflammatory markers in the brain tissues of ischaemia-induced brain-injured rats

\begin{tabular}{|c|c|c|c|c|}
\hline Sr. No. & Group & Caspase-3 (ng/mg protein) & NF-кB p65 (pg/mg protein) & TNF- $\alpha$ (ng/mg protein) \\
\hline 1 & Control & $8.17 \pm 1.12$ & $78.52 \pm 4.86$ & $127.83 \pm 6.31$ \\
\hline 2 & Ischaemic & $39.21 \pm 4.27^{\dagger \dagger}$ & $196.38 \pm 13.21^{\dagger \dagger}$ & $297.19 \pm 18.73^{\dagger \dagger}$ \\
\hline 3 & PtA & $19.63 \pm 2.19^{\star *}$ & $137.14 \pm 7.28^{\star \star *}$ & $149.50 \pm 9.75^{\star *}$ \\
\hline
\end{tabular}

TNF- $\alpha$-tumour necrosis factor $\alpha$, PtA - pseudopterosin A, data mean $\pm S D(n=10)$

${ }^{t+} p<0.01$ compared to control group; ${ }^{* *} p<0.01$ compared to the ischaemic group

A
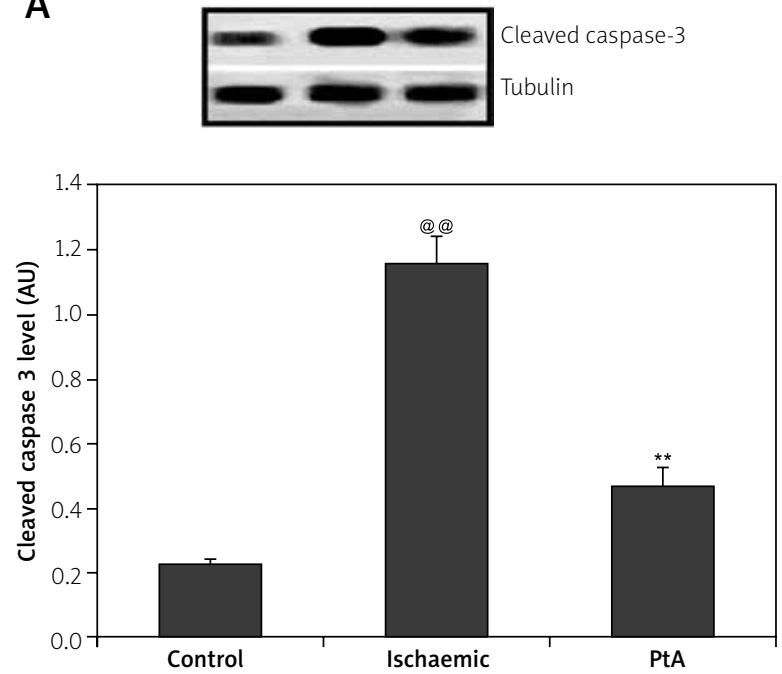

C
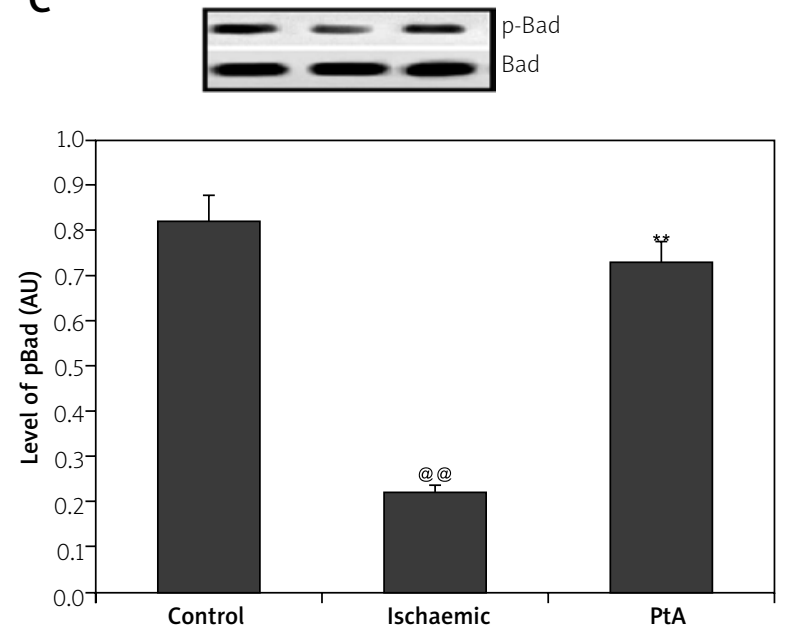

B
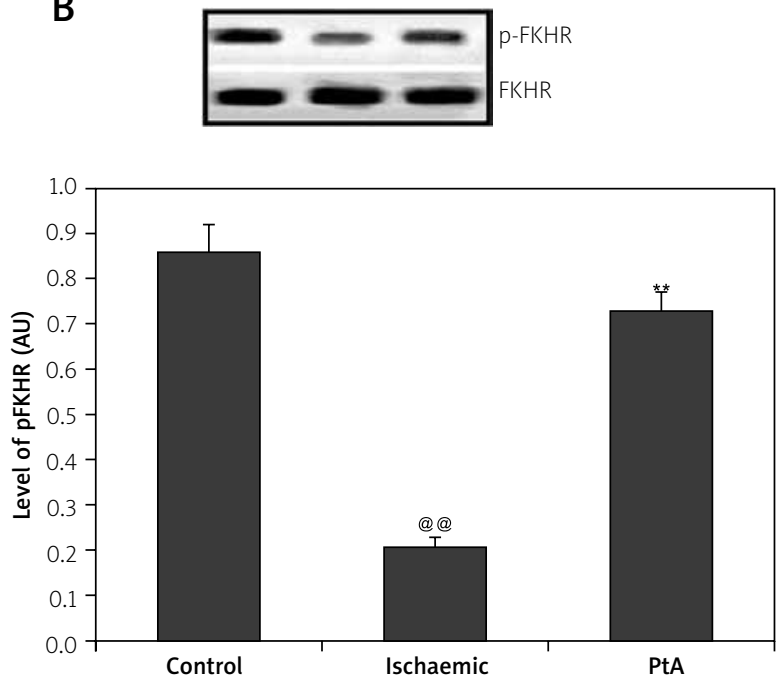

D
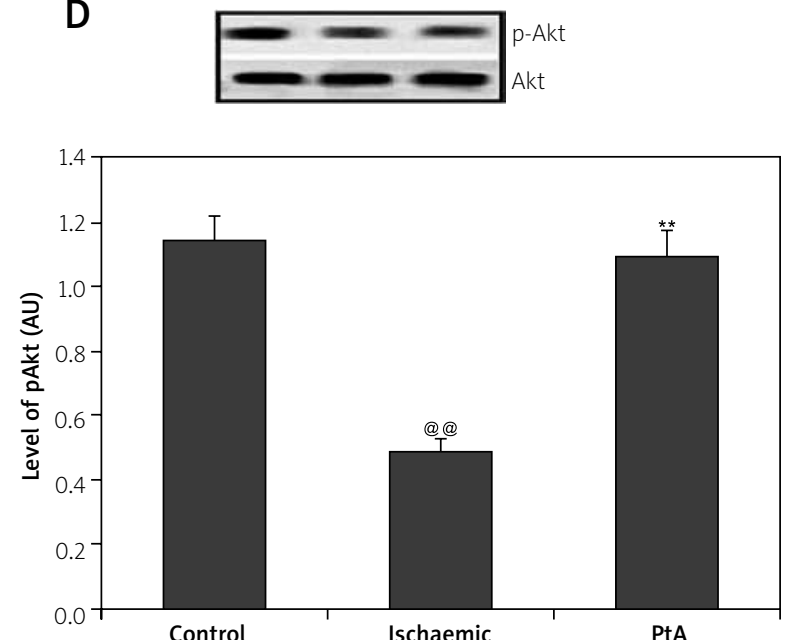

Control

Ischaemic

PtA

Fig. 4. Effect of pseudopterosin A (PtA) on the expressions of A) caspase-3, B) FKHR, C) Bad, and D) Akt in the brain tissues of ischaemia-induced brain-injured rats

caspase-3 enzyme and a decrease in the level of FKHR, Bad, and Akt in the brain tissues of the ischaemic group of rats compared to the control group. However, expressions of caspase- 3 enzyme was found to be significantly reduced and the level of FKHR, Bad, and Akt significantly enhanced in the brain tissues of the PtA-treated group of rats compared to the ischaemic group. 

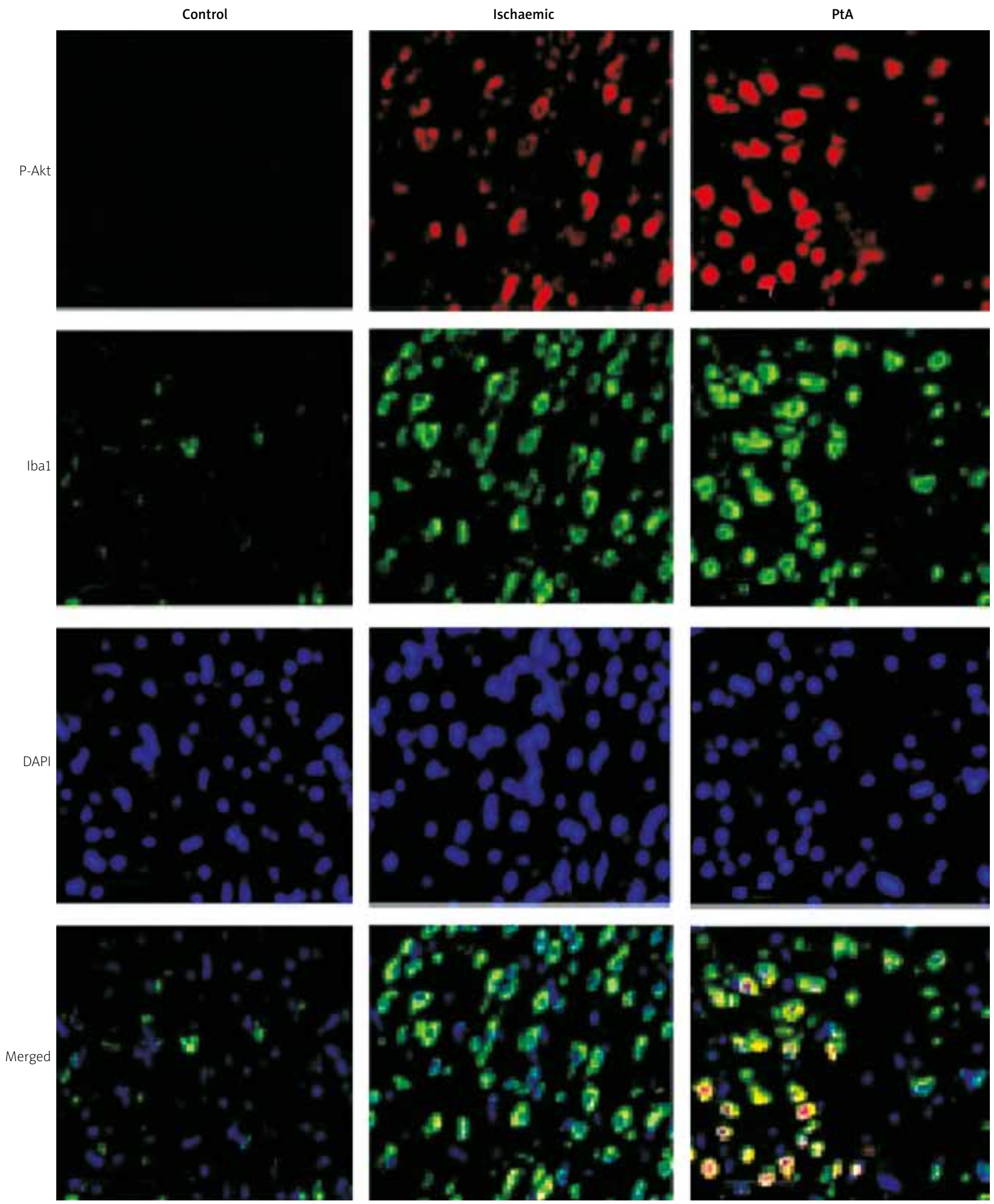

Fig. 5. Effect of PtA on the Akt phosphorylation in the brain tissues of ischaemia-induced brain-injured rats. 


\section{The effect of pseudopterosin A on Akt phosphorylation}

The effect of PtA on Akt phosphorylation in the brain tissues of ischaemia-induced brain-injured rats is shown in Figure 5. Immunohistochemical staining was done for the estimation of phosphorylation of Akt in the brain tissues of ischaemia-induced brain-injured rats. There was significant decrease in the phosphorylation of Akt in the ischaemic group compared to the control group of rats. However, treatment with PtA significantly attenuated the phosphorylation of Akt in the TS of brain tissues of ischaemia-induced brain-injured rats. Data from the western blot study also supports the results of immunohistochemical analysis.

\section{Discussion}

The present study evaluates the neuroprotective effect of PtA against brain injury and also postulates the possible mechanism of action. Drugs having anti-apoptosis and anti-inflammatory properties play a significant role in the management of brain injury [6]. Literature suggests that the level of inflammatory mediators in ischaemia-induced cerebral injured tissues [7]. In brain injury induced by ischaemia, inhibition of inflammatory mediators protects the neuronal damage. Pseudopterosin A has been reported to possess strong anti-inflammatory properties [5]. In this report inflammatory mediators such as TNF- $\alpha$ and NF- $\mathrm{BB}$ p 65 were found to be significantly reduced in the PtA-treated group compared to the ischaemic group.

In ischaemia generation of free radicals enhanced causes increase in oxidative stress, and the level of antioxidant enzymes was also reduced in ischaemic conditions [2]. Data from the present study reveal that the level of antioxidant enzyme was significantly enhanced in the PtA-treated group compared to the ischaemic group of rats.

In several neurological disorders including ischaemia induced cerebral injury up regulation of apoptosis that causes neurodegeneration [22]. In the up regulation of the process of apoptosis the family of caspase enzymes plays an important role. The activity of caspase-3 enzymes reported to enhance that upregulates the process of apoptosis. The results of the study show that PtA significantly reduces the activity of caspase- 3 enzyme and thereby attenuates the process of apoptosis [1]. The process of apoptosis is stimulated in the neuronal tissues due to enhanced level of oxidative stress and inflammatory mediators. The data from our report reveal that PtA significantly attenuates oxidative stress and inflammatory mediators and thereby reduces the process of apoptosis. Apoptosis of neuronal cells is directly related to the autophagy of cells and the Akt signalling pathway. The literature reveals that autophagy in neuronal cells is protected by inducing the Akt signalling pathway [21].

Cell death was attenuated through PI3-K/Akt signalling pathway by acting on the caspase-9, forkhead transcription factors, and Bad-like targets [19]. Survival of cells is enhanced for the phosphorylation of FKHR, Bad, and Akt. In brain injury the level of pFKHR, pBad, and pAkt was down regulated in the brain tissues, while treatment with PtA significantly enhanced the level of protein in brain tissue and thereby protects the neuronal damage.

\section{Conclusions}

The present study concludes that pseudopterosin A attenuates the neuronal injury in PMCAO model by acting on Akt signalling pathway. Pseudopterosin A down regulates the process of neuronal apoptosis by reducing the activity of caspase enzyme, and the level of oxidative stress and inflammatory mediators. The data from this report reveal that PtA has a strong neuroprotective effect and could be used clinically.

\section{Acknowledgements}

All the authors of this manuscript are thankful to the Second Military Medical University affiliated with Changzheng Hospital, China for providing the necessary facility for the conduct of the given project.

\section{Disclosure}

The authors declare no conflict of interest.

\section{References}

1. Alberts B, Johnson A, Lewis J, Raff M, Roberts K, Walter P. Molecular Biology of the Cell. 4th edition. Programmed Cell Death (Apoptosis). Garland Science, New York 2002.

2. Bhattacharyya A, Chattopadhyay R, Mitra S, Crowe SE. Oxidative stress: an essential factor in the pathogenesis of gastrointestinal mucosal diseases. Physiol Rev 2014; 94: 329-354.

3. Caplan SL, Zheng B, Scully KD, White CA, West LM. Pseudopterosin A: protection of synaptic function and potential as a neuromodulatory agent. Mar Drugs 2016; 14: E55. 
4. Chin JH, Vora N. The global burden of neurologic diseases. Neurology 2014; 83: 349-351.

5. González Y, Mendoza DT, Jones GE, Fernandez PL, Marine diterpenoids as potential anti-inflammatory agents. Mediators Inflamm 2015; 2015: 263543.

6. Hoffer BJ, Pick CG, Hoffer ME, Becker RE, Chiang YH, Greig NH. Repositioning drugs for traumatic brain injury - N-acetyl cysteine and Phenserine. J Biomed Sci 2017; 24: 71.

7. Jin R, Yang G, Li G. Inflammatory mechanisms in ischemic stroke: role of inflammatory cells. J Leukoc Biol 2010; 87: 779-789.

8. Kohl AC, Kerr RG. Pseudopterosin biosynthesis: aromatization of the diterpene cyclase product, Elisabethatriene. Mar Drugs 2003; 1: 54-65.

9. Kohl AC, Kerr RG. Identification and characterization of the pseudopterosin diterpene cyclase, elisabethatriene synthase, from the marine gorgonian, Pseudopterogorgia elisabethae. Arch Biochem Biophys 2004; 424: 97-104.

10. Kong X, Gong S, Su L, Li C, Kong Y. Neuroprotective effects of allicin on ischemia-reperfusion brain injury. Oncotarget 2017; 8: 104492-104507.

11. Li X, Zhang J, Zhu X, Wang P, Wang X, Li D. Progesterone reduces inflammation and apoptosis in neonatal rats with hypoxic ischemic brain damage through the PI3K/Akt pathway. Int J Clin Exp Med 2015; 8: 8197-8203.

12. Liu S, Sun Z, Chu P, Li H, Ahsan A, Zhou Z, Zhang Z, Sun B, Wu J, Xi Y, Han G, Lin Y, Peng J, Tang Z. EGCG protects against homocysteine-induced human umbilical vein endothelial cells apoptosis by modulating mitochondrial-dependent apoptotic signaling and PI3K/Akt/eNOS signaling pathways. Apoptosis 2017; 22: 672-680.

13. Malve $\mathrm{H}$. Exploring the ocean for new drug developments: Marine pharmacology J Pharm Bioallied Sci 2016; 8: 83-91.

14. McCulloch MWB, Haltli B, Marchbank DH, Kerr RG. Evaluation of pseudopteroxazole and pseudopterosin derivatives against Mycobacterium tuberculosis and other pathogens. Mar Drugs 2012; 10: 1711-1728.

15. Moya CE, Jacobs RS. Pseudopterosins appear to enhance the wound healing process through an adenosine receptor mediated mechanism. FASEB J 2008; 22 Suppl 1: 729.7-729.7.

16. Nour M, Scalzo F, Liebeskind DS. Ischemia-reperfusion injury in stroke. Interv Neurol 2013; 1: 185-199.

17. Pap M, Cooper GM. Role of translation initiation factor $2 B$ in control of cell survival by the phosphatidylinositol 3-kinase/ Akt/glycogen synthase kinase $3 \beta$ signaling pathway. Mol Cell Biol 2002; 22: 578-586.

18. Quillinan N, Herson PS, Traystman RJ. Neuropathophysiology of brain injury. Anesthesiol Clin 2016; 34: 453-464.

19. Sabirzhanov B, Stoica BA, Zhao Z, Loane DJ, Wu J, Dorsey SG, Faden Al. miR-711 upregulation induces neuronal cell death after traumatic brain injury. Cell Death Differ 2016; 23: 654-668.

20. Sperlich J, Kerr R, Teusch N, The marine natural product pseudopterosin blocks cytokine release of triple-negative breast cancer and monocytic leukemia cells by inhibiting NF- $\mathrm{\kappa B}$ signalling. Mar Drugs 2017; 15: 262.

21. Wang Z, Zhou L, Zheng X, Chen G, Pan R, Li J, Liu W. Autophagy protects against PI3K/Akt/mTOR-mediated apoptosis of spinal cord neurons after mechanical injury. Neurosci Lett 2017; 656: 158-164.
22. Xu M, Zhang H. Death and survival of neuronal and astrocytic cells in ischemic brain injury: a role of autophagy. Acta Pharmacol Sin 2011; 32: 1089-1099. 